\title{
Some inequalities related to strong convergence of Riesz logarithmic means
}

\author{
D. Lukkassen' ${ }^{1}$ L.E. Persson ${ }^{1,2^{*}}$, G. Tephnadze ${ }^{3}$ and G. Tutberidze ${ }^{4,1}$
}

"Correspondence:
larserik6pers@gmail.com
${ }^{1}$ UiT The Arctic University of
Norway, Narvik, Norway
${ }^{2}$ Department of Mathematics and
Computer Science, Karlstad
University, Karlstad, Sweden
Full list of author information is
available at the end of the article

available at the end of the article

\begin{abstract}
In this paper we derive a new strong convergence theorem of Riesz logarithmic means of the one-dimensional Vilenkin-Fourier (Walsh-Fourier) series. The corresponding inequality is pointed out and it is also proved that the inequality is in a sense sharp, at least for the case with Walsh-Fourier series.
\end{abstract}

MSC: 26D10; 26D20;42B25; 42C10

Keywords: Inequalities; Vilenkin systems; Walsh system; Riesz logarithmic means; Martingale Hardy space; Strong convergence

\section{Introduction}

Concerning definitions used in this introduction we refer to Sect. 2. Weisz [47] proved the boundedness of the maximal operator of Fejér means $\sigma^{\psi, *}$ with respect to bounded Vilenkin systems from the martingale Hardy space $H_{p}\left(G_{m}\right)$ to the space $L_{p}\left(G_{m}\right)$, for $p>1 / 2$. Simon [31] gave a counterexample, which shows that boundedness does not hold for $0<$ $p<1 / 2$. The corresponding counterexample for $p=1 / 2$ is due to Goginava [14]. Moreover, Weisz [50] proved the following result.

Theorem W The maximal operator of Fejér means $\sigma^{\psi, *}$ is bounded from the Hardy space $H_{1 / 2}\left(G_{m}\right)$ to the space weak- $L_{1 / 2}\left(G_{m}\right)$.

In [35] and [36] it was proved that the maximal operator $\widetilde{\sigma}_{p}^{\psi, *}$ defined by

$$
\widetilde{\sigma}_{p}^{\psi, *}:=\sup _{n \in \mathbb{N}} \frac{\left|\sigma_{n}^{\psi}\right|}{(n+1)^{1 / p-2} \log ^{2[1 / 2+p]}(n+1)}
$$

where $0<p \leq 1 / 2$ and $[1 / 2+p]$ denotes the integer part of $1 / 2+p$, is bounded from the Hardy space $H_{p}\left(G_{m}\right)$ to the space $L_{p}\left(G_{m}\right)$. Moreover, for any nondecreasing function $\varphi$ : $\mathbb{N}_{+} \rightarrow[1, \infty)$ satisfying the condition

$$
\varlimsup_{n \rightarrow \infty} \frac{(n+1)^{1 / p-2} \log ^{2[1 / 2+p]}(n+1)}{\varphi(n)}=+\infty,
$$

(c) The Author(s) 2020. This article is licensed under a Creative Commons Attribution 4.0 International License, which permits use, sharing, adaptation, distribution and reproduction in any medium or format, as long as you give appropriate credit to the original author(s) and the source, provide a link to the Creative Commons licence, and indicate if changes were made. The images or other third party material in this article are included in the article's Creative Commons licence, unless indicated otherwise in a credit line to the material. If material is not included in the article's Creative Commons licence and your intended use is not permitted by statutory regulation or exceeds the permitted use, you will need to obtain permission directly from the copyright holder. To view a copy of this licence, visit http://creativecommons.org/licenses/by/4.0/. 
there exists a martingale $f \in H_{p}\left(G_{m}\right)$, such that

$$
\sup _{n \in \mathbb{N}}\left\|\frac{\sigma_{n}^{\psi} f}{\varphi(n)}\right\|_{p}=\infty
$$

For Walsh-Kaczmarzi system some analogical results were proved in [16] and [37].

Weisz [47] considered the norm convergence of the Fejér means of a Vilenkin-Fourier series and proved the following result.

Theorem W1 (Weisz) Let $p>1 / 2$ and $f \in H_{p}\left(G_{m}\right)$. Then there exists an absolute constant $c_{p}$, depending only on $p$, such that for all $k=1,2, \ldots$ and $f \in H_{p}\left(G_{m}\right)$ the following inequality holds:

$$
\left\|\sigma_{k}^{\psi} f\right\|_{p} \leq c_{p}\|f\|_{H_{p}\left(G_{m}\right)}
$$

Moreover, in [34] it was proved that the assumption $p>1 / 2$ in Theorem W1 is essential. In fact, the following is true.

Theorem T1 There exists a martingale $f \in H_{1 / 2}\left(G_{m}\right)$ such that

$$
\sup _{n \in \mathbb{N}}\left\|\sigma_{n}^{\psi} f\right\|_{1 / 2}=+\infty
$$

Theorem W1 implies that

$$
\frac{1}{n^{2 p-1}} \sum_{k=1}^{n} \frac{\left\|\sigma_{k}^{\psi} f\right\|_{p}^{p}}{k^{2-2 p}} \leq c_{p}\|f\|_{H_{p}\left(G_{m}\right)}^{p}, \quad 1 / 2<p<\infty, n=1,2, \ldots
$$

If Theorem W1 holds for $0<p \leq 1 / 2$, then we would have

$$
\frac{1}{\log ^{[1 / 2+p]} n} \sum_{k=1}^{n} \frac{\left\|\sigma_{k}^{\psi} f\right\|_{p}^{p}}{k^{2-2 p}} \leq c_{p}\|f\|_{H_{p}\left(G_{m}\right)}^{p}, \quad 0<p \leq 1 / 2, n=2,3, \ldots
$$

For the Walsh system in [38] and for the bounded Vilenkin systems in [37] were proved that (1.2) holds, though Theorem T1 is not true for $0<p<1 / 2$.

Some results concerning summability of the Fejér means of a Vilenkin-Fourier series can be found in $[10,12,16,25,28,30]$.

The Riesz logarithmic means with respect to the Walsh system was studied by Simon [31], Goginava [15], Gát, Nagy [13] and for Vilenkin systems by Gát [11] and Blahota, Gát [3], Persson, Ragusa, Samko, Wall [26]. Moreover, in [27] it was proved that the maximal operator of the Riesz logarithmic means of a Vilenkin-Fourier series is bounded from the martingale Hardy space $H_{p}\left(G_{m}\right)$ to the space $L_{p}\left(G_{m}\right)$ when $p>1 / 2$ and is not bounded from the martingale Hardy space $H_{p}\left(G_{m}\right)$ to the space $L_{p}\left(G_{m}\right)$ when $0<p \leq 1 / 2$.

In [35] and [36] it was proved that the Riesz logarithmic means has better properties than the Fejér means. In particular, one considered the maximal operator $\widetilde{R}_{p}^{\psi, *}$ of a Riesz logarithmic means $\widetilde{R}_{p}^{\psi, *}$ defined by

$$
\widetilde{R}_{p}^{\psi, *}:=\sup _{n \in \mathbb{N}} \frac{\left|R_{n}^{\psi}\right| \log (n+1)}{(n+1)^{1 / p-2} \log ^{2[1 / 2+p]}(n+1)}
$$


where $0<p \leq 1 / 2$ and $[1 / 2+p]$ denotes the integer part of $1 / 2+p$, which is bounded from the Hardy space $H_{p}\left(G_{m}\right)$ to the space $L_{p}\left(G_{m}\right)$.

Moreover, this result is sharp in the following sense: For any nondecreasing function $\varphi: \mathbb{N}_{+} \rightarrow[1, \infty)$ satisfying the condition

$$
\varlimsup_{n \rightarrow \infty} \frac{(n+1)^{1 / p-2} \log ^{2[1 / 2+p]}(n+1)}{\varphi(n) \log (n+1)}=\infty,
$$

there exists a martingale $f \in H_{p}\left(G_{m}\right)$, such that

$$
\sup _{n \in \mathbb{N}}\left\|\frac{R_{n}^{\psi} f}{\varphi(n)}\right\|_{p}=\infty
$$

The main aim of this paper is to derive a new strong convergence theorem of the Riesz logarithmic means of one-dimensional Vilenkin-Fourier (Walsh-Fourier) series (see Theorem 1). The corresponding inequality is pointed out. The sharpness is proved in Theorem 2, at least for the case with Walsh-Fourier series.

The paper is organized as follows: In Sect. 2 some definitions and notations are presented. The main results are presented and proved in Sect. 3. Section 4 is reserved for some concluding remarks and open problems.

\section{Definitions and notations}

Let $\mathbb{N}_{+}$denote the set of positive integers, $\mathbb{N}:=\mathbb{N}_{+} \cup\{0\}$.

Let $m:=\left(m_{0}, m_{1}, \ldots\right)$ denote a sequence of positive integers not less than 2 .

Denote by

$$
Z_{m_{k}}:=\left\{0,1, \ldots m_{k}-1\right\}
$$

the additive group of integers modulo $m_{k}$.

Define the group $G_{m}$ as the complete direct product of the group $Z_{m_{j}}$ with the product of the discrete topologies of the $Z_{m_{j}}$.

The direct product $\mu$ of the measures

$$
\mu_{k}(\{j\}):=1 / m_{k} \quad\left(j \in Z_{m_{k}}\right)
$$

is a Haar measure on $G_{m}$ with $\mu\left(G_{m}\right)=1$.

If $\sup _{n \in \mathbb{N}} m_{n}<\infty$, then we call $G_{m}$ a bounded Vilenkin group. If the generating sequence $m$ is not bounded, then $G_{m}$ is said to be an unbounded Vilenkin group. In this paper we discuss only bounded Vilenkin groups.

The elements of $G_{m}$ are represented by the sequences

$$
x:=\left(x_{0}, x_{1}, \ldots, x_{j}, \ldots\right) \quad\left(x_{k} \in Z_{m_{k}}\right) .
$$

It is easy to give a base for the neighborhood of $G_{m}$, namely

$$
\begin{aligned}
& I_{0}(x):=G_{m}, \\
& I_{n}(x):=\left\{y \in G_{m} \mid y_{0}=x_{0}, \ldots y_{n-1}=x_{n-1}\right\} \quad\left(x \in G_{m}, n \in \mathbb{N}\right) .
\end{aligned}
$$


Denote $I_{n}:=I_{n}(0)$ for $n \in \mathbb{N}$ and $\overline{I_{n}}:=G_{m} \backslash I_{n}$.

Let

$$
e_{n}:=\left(0,0, \ldots, x_{n}=1,0, \ldots\right) \in G_{m} \quad(n \in \mathbb{N})
$$

It is evident that

$$
\overline{I_{M}}=\left(\bigcup_{k=0}^{M-2} \bigcup_{x_{k}=1}^{m_{k}-1} \bigcup_{l=k+1}^{M-1} \bigcup_{x_{l}=1}^{m_{l}-1} I_{l+1}\left(x_{k} e_{k}+x_{l} e_{l}\right)\right) \cup\left(\bigcup_{k=1}^{M-1} \bigcup_{x_{k}=1}^{m_{k}-1} I_{M}\left(x_{k} e_{k}\right)\right)
$$

If we define the so-called generalized number system based on $m$ in the following way:

$$
M_{0}:=1, \quad M_{k+1}:=m_{k} M_{k} \quad(k \in \mathbb{N}),
$$

then every $n \in \mathbb{N}$ can be uniquely expressed as $n=\sum_{k=0}^{\infty} n_{j} M_{j}$, where $n_{j} \in Z_{m_{j}}(j \in \mathbb{N})$ and only a finite number of the $n_{j}$ differ from zero. Let $|n|:=\max \left\{j \in \mathbb{N} ; n_{j} \neq 0\right\}$.

The norm (or quasi-norm when $p<1$ ) of the space $L_{p}\left(G_{m}\right)$ is defined by

$$
\|f\|_{p}:=\left(\int_{G_{m}}|f|^{p} d \mu\right)^{1 / p} \quad(0<p<\infty) .
$$

The space weak- $L_{p}\left(G_{m}\right)$ consists of all measurable functions $f$ for which

$$
\|f\|_{\text {weak }-L_{p}\left(G_{m}\right)}:=\sup _{\lambda>0} \lambda^{p} \mu(f>\lambda)<+\infty \text {. }
$$

Next, we introduce on $G_{m}$ an orthonormal system which is called the Vilenkin system. Let us define complex valued function $r_{k}(x): G_{m} \rightarrow \mathbb{C}$, the generalized Rademacher functions, as

$$
r_{k}(x):=\exp \left(2 \pi i x_{k} / m_{k}\right) \quad\left(i^{2}=-1, x \in G_{m}, k \in \mathbb{N}\right)
$$

Now, define the Vilenkin system $\psi:=\left(\psi_{n}: n \in \mathbb{N}\right)$ on $G_{m}$ as

$$
\psi_{n}(x):=\prod_{k=0}^{\infty} r_{k}^{n_{k}}(x) \quad(n \in \mathbb{N})
$$

The Vilenkin systems are orthonormal and complete in $L_{2}\left(G_{m}\right)$ (for details see e.g. [1]). Specifically, we call this system Walsh-Paley if $m_{k}=2$, for all $k \in \mathbb{N}$. In this case we have the dyadic group $G_{2}=\prod_{j=0}^{\infty} Z_{2}$, which is called the Walsh group and the Vilenkin system coincides with the Walsh functions defined by (for details see e.g. [17] and [29])

$$
w_{n}(x):=\prod_{k=0}^{\infty} r_{k}^{n_{k}}(x)=r_{|n|}(x)(-1)^{\sum_{k=0}^{|n|-1} n_{k} x_{k}} \quad(n \in \mathbb{N})
$$

where $n_{k}=0 \vee 1$ and $x_{k}=0 \vee 1$.

Now, we introduce analogues of the usual definitions in Fourier analysis. 
If $f \in L_{1}\left(G_{m}\right)$, then we can establish the Fourier coefficients, the partial sums of the Fourier series, the Fejér means, the Dirichlet and Fejér kernels with respect to the Vilenkin system $\psi$ (Walsh system $w$ ) in the usual manner:

$$
\begin{aligned}
\widehat{f}^{\alpha}(k):=\int_{G_{m}} f \bar{\alpha}_{k} d \mu \quad\left(\alpha_{k}=w_{k} \text { or } \psi_{k}\right)(k \in \mathbb{N}), \\
S_{n}^{\alpha} f:=\sum_{k=0}^{n-1} \widehat{f}(k) \alpha_{k} \quad\left(\alpha_{k}=w_{k} \text { or } \psi_{k}\right)\left(n \in \mathbb{N}_{+}, S_{0}^{\alpha} f:=0\right), \\
\sigma_{n}^{\alpha} f:=\frac{1}{n} \sum_{k=0}^{n-1} S_{k}^{\alpha} f \quad(\alpha=w \text { or } \psi)\left(n \in \mathbb{N}_{+}\right), \\
D_{n}^{\alpha}:=\sum_{k=0}^{n-1} \alpha_{k} \quad(\alpha=w \text { or } \psi)\left(n \in \mathbb{N}_{+}\right), \\
K_{n}^{\alpha}:=\frac{1}{n} \sum_{k=0}^{n-1} D_{k}^{\alpha} \quad(\alpha=w \text { or } \psi)\left(n \in \mathbb{N}_{+}\right) .
\end{aligned}
$$

It is well known that (see e.g. [1])

$$
\sup _{n \in \mathbb{N}} \int_{G_{m}}\left|K_{n}^{\alpha}\right| d \mu \leq c<\infty, \quad \text { where } \alpha=w \text { or } \psi
$$

The $\sigma$-algebra generated by the intervals $\left\{I_{n}(x): x \in G_{m}\right\}$ will be denoted by $\digamma_{n}(n \in \mathbb{N})$. Denote by $f=\left(f^{(n)}, n \in \mathbb{N}\right.$ ) a martingale with respect to $\digamma_{n}(n \in \mathbb{N}$ ) (for details see e.g. $[5,23,46])$. The maximal function of a martingale $f$ is defend by

$$
f^{*}=\sup _{n \in \mathbb{N}}\left|f^{(n)}\right| .
$$

In the case $f \in L_{1}\left(G_{m}\right)$, the maximal functions are also given by

$$
f^{*}(x)=\sup _{n \in \mathbb{N}} \frac{1}{\left|I_{n}(x)\right|}\left|\int_{I_{n}(x)} f(u) \mu(u)\right|
$$

For $0<p<\infty$ the Hardy martingale spaces $H_{p}\left(G_{m}\right)$ consist of all martingales for which

$$
\|f\|_{H_{p}\left(G_{m}\right)}:=\left\|f^{*}\right\|_{p}<\infty
$$

If $f \in L_{1}\left(G_{m}\right)$, then it is easy to show that $S_{M_{n}} f$ is $\digamma_{n}$ measurable and the sequence $\left(S_{M_{n}} f: n \in \mathbb{N}\right)$ is a martingale. If $f=\left(f^{(n)}, n \in \mathbb{N}\right)$ is a martingale, then the Vilenkin-Fourier (Walsh-Fourier) coefficients must be defined in a slightly different manner, namely

$$
\widehat{f}(i):=\lim _{k \rightarrow \infty} \int_{G_{m}} f^{(k)}(x) \bar{\alpha}_{i}(x) d \mu(x), \quad \text { where } \alpha=w \text { or } \psi
$$

The Vilenkin-Fourier coefficients of $f \in L_{1}\left(G_{m}\right)$ are the same as those of the martingale $\left(S_{M_{n}} f: n \in \mathbb{N}\right)$ obtained from $f$. 
In the literature, there is the notion of the Riesz logarithmic means of a Fourier series. The $n$th Riesz logarithmic means of the Fourier series of an integrable function $f$ is defined by

$$
R_{n}^{\alpha} f:=\frac{1}{l_{n}} \sum_{k=1}^{n} \frac{S_{k}^{\alpha} f}{k}, \quad \text { where } \alpha=w \text { or } \psi,
$$

with

$$
l_{n}:=\sum_{k=1}^{n} \frac{1}{k}
$$

The kernels of Riesz's logarithmic means are defined by

$$
L_{n}^{\alpha}:=\frac{1}{l_{n}} \sum_{k=1}^{n} \frac{D_{k}^{\alpha}}{k}, \quad \text { where }(\alpha=w \text { or } \psi) .
$$

For the martingale $f$ we consider the following maximal operators:

$$
\begin{aligned}
& \sigma^{\alpha, *} f: \sup _{n \in \mathbb{N}}\left|\sigma_{n}^{\alpha} f\right| \quad(\alpha=w \text { or } \psi), \\
& R^{*} f:=\sup _{n \in \mathbb{N}}\left|R_{n}^{\alpha} f\right| \quad(\alpha=w \text { or } \psi), \\
& \widetilde{R}^{\alpha, *} f:=\sup _{n \in \mathbb{N}} \frac{\left|R_{n}^{\alpha} f\right|}{\log (n+1)} \quad(\alpha=w \text { or } \psi), \\
& \widetilde{R}_{p}^{\alpha, *} f:=\sup _{n \in \mathbb{N}} \frac{\log (n+1)\left|R_{n}^{\alpha} f\right|}{(n+1)^{1 / p-2}} \quad(\alpha=w \text { or } \psi) .
\end{aligned}
$$

A bounded measurable function $a$ is a $p$-atom, if there exists an interval $I$, such that

$$
\int_{I} a d \mu=0, \quad\|a\|_{\infty} \leq \mu(I)^{-1 / p}, \quad \operatorname{supp}(a) \subset I .
$$

In order to prove our main results we need the following lemma of Weisz (for details see e.g. Weisz [49]).

Proposition 1 A martingale $f=\left(f^{(n)}, n \in \mathbb{N}\right)$ is in $H_{p}\left(G_{m}\right)(0<p \leq 1)$ if and only if there exist a sequence $\left(a_{k}, k \in \mathbb{N}\right)$ of $p$-atoms and a sequence $\left(\mu_{k}, k \in \mathbb{N}\right)$ of a real numbers such that for every $n \in \mathbb{N}$

$$
\sum_{k=0}^{\infty} \mu_{k} S_{M_{n}} a_{k}=f^{(n)}
$$

and

$$
\sum_{k=0}^{\infty}\left|\mu_{k}\right|^{p}<\infty
$$

Moreover, $\|f\|_{H_{p}\left(G_{m}\right)} \backsim \inf \left(\sum_{k=0}^{\infty}\left|\mu_{k}\right|^{p}\right)^{1 / p}$, where the infimum is taken over all decompositions off of the form (2.3). 
By using atomic characterization (see Proposition 1) it can be easily proved that the following statement holds (see e.g. Weisz [50]).

Proposition 2 Suppose that an operator $T$ is sub-linear and for some $0<p_{0} \leq 1$

$$
\int_{\bar{I}}|T a|^{p_{0}} d \mu \leq c_{p}<\infty
$$

for every $p_{0}$-atom $a$, where I denotes the support of the atom. If $T$ is bounded from $L_{p_{1}}$ to $L_{p_{1}}\left(1<p_{1} \leq \infty\right)$, then

$$
\|T f\|_{p_{0}} \leq c_{p_{0}}\|f\|_{H_{p_{0}}\left(G_{m}\right)}
$$

Let us define classical Hardy spaces (see e.g. [44]). Let $H_{p}(D), p>0$ be the onedimensional complex quasi-Banach space of analytic functions $f$ on the unit disc $D:=$ $(z:|z|<1)$ for which

$$
\|f\|_{H_{p}(D)}=\sup _{r<1} \frac{1}{2 \pi}\left(\int_{[-\pi, \pi]}\left|f\left(r e^{i t}\right)\right|^{p} d t\right)^{1 / p} .
$$

Now, we define real Hardy spaces. A real-valued distributions $f(t) \in D^{\prime}(T)$ belongs to $H_{p}(T)$ where $T=(-\pi, \pi]$ if and only if there exists a function $F(z) \in H_{p}(D)$ with the properties $\operatorname{Im}(F(0))=0$ and $f(t)=\lim _{r \rightarrow 1} \operatorname{Re} F\left(r e^{i t}\right)$ in the sense of distributions. Equipped with quasi-norm $\|f(z)\|_{H_{p}(T)}=\|F(z)\|_{H_{p}(D)}$ the class obviously becomes a real quasi-Banach space with quite the same properties as $H_{p}(D)$. Atomic decomposition of classical Hardy spaces and real Hardy spaces can be found e.g. in Fefferman and Stein [6] (see also Later [19], Torchinsky [44], Wilson [51]).

\section{Main results}

Our first main result reads as follows.

Theorem 1 Let $0<p<1 / 2$ and $f \in H_{p}\left(G_{m}\right)$. Then there exists an absolute constant $c_{p}$, depending only on $p$, such that the inequality

$$
\sum_{n=1}^{\infty} \frac{\log ^{p} n\left\|R_{n}^{\psi} f\right\|_{H_{p}\left(G_{m}\right)}^{p}}{n^{2-2 p}} \leq c_{p}\|f\|_{H_{p}\left(G_{m}\right)}^{p}
$$

holds, where $R_{n}^{\psi} f$ denotes the nth Riesz logarithmic mean with respect to the VilenkinFourier series off.

For the proof of Theorem 1 we will use the following lemmas.

Lemma 1 (see [38]) Let $x \in I_{N}\left(x_{k} e_{k}+x_{l} e_{l}\right), 1 \leq x_{k} \leq m_{k}-1,1 \leq x_{l} \leq m_{l}-1, k=0, \ldots, N-2$, $l=k+1, \ldots, N-1$. Then

$$
\int_{I_{N}}\left|K_{n}^{\psi}(x-t)\right| d \mu(t) \leq \frac{c M_{l} M_{k}}{n M_{N}}, \quad \text { when } n \geq M_{N}
$$


Let $x \in I_{N}\left(x_{k} e_{k}\right), 1 \leq x_{k} \leq m_{k}-1, k=0, \ldots, N-1$. Then

$$
\int_{I_{N}}\left|K_{n}^{\psi}(x-t)\right| d \mu(t) \leq \frac{c M_{k}}{M_{N}}, \quad \text { when } n \geq M_{N}
$$

Lemma 2 (see [39]) Let $x \in I_{N}\left(x_{k} e_{k}+x_{l} e_{l}\right), 1 \leq x_{k} \leq m_{k}-1,1 \leq x_{l} \leq m_{l}-1, k=0, \ldots, N-2$, $l=k+1, \ldots, N-1$. Then

$$
\int_{I_{N}} \sum_{j=M_{N}+1}^{n} \frac{\left|K_{j}^{\psi}(x-t)\right|}{j+1} d \mu(t) \leq \frac{c M_{k} M_{l}}{M_{N}^{2}}
$$

Let $x \in I_{N}\left(x_{k} e_{k}\right), 1 \leq x_{k} \leq m_{k}-1, k=0, \ldots, N-1$. Then

$$
\int_{I_{N}} \sum_{j=M_{N}+1}^{n} \frac{\left|K_{j}^{\psi}(x-t)\right|}{j+1} d \mu(t) \leq \frac{c M_{k}}{M_{N}} l_{n}
$$

Proof By using an Abel transformation, the kernels of the Riesz logarithmic means can be rewritten as (see also [39])

$$
L_{n}^{\psi}=\frac{1}{l_{n}} \sum_{j=1}^{n-1} \frac{K_{j}^{\psi}}{j+1}+\frac{K_{n}^{\psi}}{l_{n}}
$$

Hence, according to (2.2) we get

$$
\sup _{n \in \mathbb{N}} \int_{G_{m}}\left|L_{n}^{\alpha}\right| d \mu \leq c<\infty, \quad \text { where } \alpha=w \text { or } \psi
$$

and it follows that $R_{n}^{\psi}$ is bounded from $L_{\infty}$ to $L_{\infty}$. By Proposition 2 , the proof of Theorem 1 will be complete, if we show that

$$
\sum_{n=1}^{\infty} \frac{\log ^{p} n \int_{\bar{I}}\left|R_{n}^{\psi} a\right|^{p} d \mu}{n^{2-2 p}} \leq c_{p}<\infty, \quad \text { for } 0<p<1 / 2
$$

for every $p$-atom $a$, where $I$ denotes the support of the atom.

Let $a$ be an arbitrary p-atom with support $I$ and $\mu(I)=M_{N}^{-1}$. We may assume that $I=I_{N}$. It is easy to see that $R_{n}^{\psi} a=\sigma_{n}^{\psi}(a)=0$, when $n \leq M_{N}$. Therefore we suppose that $n>M_{N}$.

Since $\|a\|_{\infty} \leq c M_{N}^{2}$ if we apply (3.2), then we can conclude that

$$
\begin{aligned}
& \left|R_{n}^{\psi} a(x)\right| \\
& =\int_{I_{N}}|a(t)|\left|L_{n}^{\psi}(x-t)\right| d \mu(t) \\
& \leq\|a\|_{\infty} \int_{I_{N}}\left|L_{n}^{\psi}(x-t)\right| d \mu(t) \\
& \leq \frac{c M_{N}^{1 / p}}{l_{n}} \int_{I_{N}} \sum_{j=M_{N}+1}^{n-1} \frac{\left|K_{j}^{\psi}(x-t)\right|}{j+1} d \mu(t) \\
& \quad+\frac{c M_{N}^{1 / p}}{l_{n}} \int_{I_{N}}\left|K_{n}^{\psi}(x-t)\right| d \mu(t) .
\end{aligned}
$$


Let $x \in I_{N}\left(x_{k} e_{k}+x_{l} e_{l}\right), 1 \leq x_{k} \leq m_{k}-1,1 \leq x_{l} \leq m_{l}-1, k=0, \ldots, N-2, l=k+1, \ldots, N-1$. From Lemmas 1 and 2 it follows that

$$
\left|R_{n}^{\psi} a(x)\right| \leq \frac{c M_{l} M_{k} M_{N}^{1 / p-2}}{\log (n+1)}
$$

Let $x \in I_{N}\left(x_{k} e_{k}\right), 1 \leq x_{k} \leq m_{k}-1, k=0, \ldots, N-1$. Applying Lemmas 1 and 2 we can conclude that

$$
\left|R_{n}^{\psi} a(x)\right| \leq M_{N}^{1 / p-1} M_{k}
$$

By combining (2.1) and (3.4)-(3.6) we obtain

$$
\begin{aligned}
& \int_{\overline{I_{N}}}\left|R_{n}^{\psi} a(x)\right|^{p} d \mu(x) \\
& \quad=\sum_{k=0}^{N-2} \sum_{l=k+1}^{N-1} \sum_{x_{j}=0, j \in\{l+1, \ldots, N-1}^{m_{j-1}} \int_{I_{N}^{k, l}}\left|R_{n}^{\psi} a\right|^{p} d \mu+\sum_{k=0}^{N-1} \int_{I_{N}^{k, N}}\left|R_{n}^{\psi} a\right|^{p} d \mu \\
& \quad \leq c \sum_{k=0}^{N-2} \sum_{l=k+1}^{N-1} \frac{m_{l+1} \ldots m_{N-1}}{M_{N}} \frac{\left(M_{l} M_{k}\right)^{p} M_{N}^{1-2 p}}{\log ^{p}(n+1)}+\sum_{k=0}^{N-1} \frac{1}{M_{N}} M_{k}^{p} M_{N}^{1-p} \\
& \quad \leq \frac{c M_{N}^{1-2 p}}{\log ^{p}(n+1)} \sum_{k=0}^{N-2} \sum_{l=k+1}^{N-1} \frac{\left(M_{l} M_{k}\right)^{p}}{M_{l}}+\sum_{k=0}^{N-1} \frac{M_{k}^{p}}{M_{N}^{p}} \\
& \quad \leq \frac{c M_{N}^{1-2 p}}{\log ^{p}(n+1)}+c_{p} .
\end{aligned}
$$

It is easy to see that

$$
\sum_{n=M_{N}+1}^{\infty} \frac{1}{n^{2-2 p}} \leq \frac{c}{M_{N}^{1-2 p}}, \quad \text { for } 0<p<1 / 2
$$

By combining (3.7) and (3.8) we get

$$
\begin{aligned}
& \sum_{n=M_{N}+1}^{\infty} \frac{\log ^{p} n \int_{\overline{I_{N}}}\left|R_{n} a\right|^{p} d \mu}{n^{2-2 p}} \\
& \leq \sum_{n=M_{N}+1}^{\infty}\left(\frac{c_{p} M_{N}^{1-2 p}}{n^{2-p}}+\frac{c_{p}}{n^{2-p}}\right)+c_{p} \\
& \quad \leq c_{p} M_{N}^{1-2 p} \sum_{n=M_{N}+1}^{\infty} \frac{1}{n^{2-2 p}}+\sum_{n=M_{N}+1}^{\infty} \frac{1}{n^{2-p}}+c_{p} \leq C_{p}<\infty .
\end{aligned}
$$

It means that (3.3) holds true and the proof is complete.

Our next main result shows in particular that the inequality in Theorem 1 is in a special sense sharp at least in the case of Walsh-Fourier series (cf. also Problem 2 in the next section). 
Theorem 2 Let $0<p<1 / 2$ and $\Phi: \mathbb{N} \rightarrow[1, \infty)$ be any nondecreasing function, satisfying the condition

$$
\lim _{n \rightarrow \infty} \Phi(n)=+\infty .
$$

Then there exists a martingale $f \in H_{p}\left(G_{2}\right)$ such that

$$
\sum_{n=1}^{\infty} \frac{\log ^{p} n\left\|R_{n}^{w} f\right\|_{p}^{p} \Phi(n)}{n^{2-2 p}}=\infty,
$$

where $R_{n}^{w} f$ denotes the $n$th Riesz logarithmic means with respect to Walsh-Fourier series off.

Proof It is evident that if we assume that $\Phi(n) \geq c n$, where $c$ is some positive constant then

$$
\frac{\log ^{p} n \Phi(n)}{n^{2-2 p}} \geq n^{1-2 p} \log ^{p} n \rightarrow \infty, \quad \text { as } n \rightarrow \infty,
$$

and also (3.10) holds. So, without loss of generality we may assume that there exists an increasing sequence of positive integers $\left\{\alpha_{k}^{\prime}: k \in \mathbb{N}\right\}$ such that

$$
\Phi\left(\alpha_{k}^{\prime}\right)=o\left(\alpha_{k}^{\prime}\right), \quad \text { as } k \rightarrow \infty
$$

Let $\left\{\alpha_{k}: k \in \mathbb{N}\right\} \subseteq\left\{\alpha_{k}^{\prime}: k \in \mathbb{N}\right\}$ be an increasing sequence of positive integers such that $\alpha_{0} \geq 2$ and

$$
\begin{aligned}
& \sum_{k=0}^{\infty} \frac{1}{\Phi^{1 / 2}\left(2^{2 \alpha_{k}}\right)}<\infty, \\
& \sum_{\eta=0}^{k-1} \frac{2^{2 \alpha_{\eta} / p}}{\Phi^{1 / 2 p}\left(2^{2 \alpha_{\eta}}\right)} \leq \frac{2^{2 \alpha_{k-1} / p+1}}{\Phi^{1 / 2 p}\left(2^{2 \alpha_{k-1}}\right)}, \\
& \frac{2^{2 \alpha_{k-1} / p+1}}{\Phi^{1 / 2 p}\left(2^{\left.2 \alpha_{k-1}\right)}\right.} \leq \frac{1}{128 \alpha_{k}} \frac{2^{2 \alpha_{k}(1 / p-2)}}{\Phi^{1 / 2 p}\left(2^{2 \alpha_{k}}\right)} .
\end{aligned}
$$

We note that under condition (3.11) we can conclude that

$$
\frac{2^{2 \alpha_{\eta} / p}}{\Phi^{1 / 2 p}\left(2^{2 \alpha_{\eta}}\right)} \geq\left(\frac{2^{2 \alpha_{\eta}}}{\Phi\left(2^{2 \alpha_{\eta}}\right)}\right)^{1 / 2 p} \rightarrow \infty, \quad \text { as } \eta \rightarrow \infty
$$

and it immediately follows that such an increasing sequence $\left\{\alpha_{k}: k \in \mathbb{N}\right\}$, which satisfies conditions (3.12)-(3.14), can be constructed.

Let

$$
f^{(A)}(x):=\sum_{\left\{k ; 2 \alpha_{k}<A\right\}} \lambda_{k} a_{k}
$$

where

$$
\lambda_{k}=\frac{1}{\Phi^{1 / 2 p}\left(2^{2 \alpha_{k}}\right)}
$$


and

$$
a_{k}=2^{2 \alpha_{k}(1 / p-1)}\left(D_{2^{2 \alpha_{k}+1}}-D_{2^{2 \alpha_{k}}}\right) .
$$

From (3.12) and Lemma 1 we can conclude that $f=\left(f^{(n)}, n \in \mathbb{N}\right) \in H_{p}\left(G_{2}\right)$.

It is easy to show that

$$
\widehat{f}^{w}(j)= \begin{cases}\frac{2^{2 \alpha_{k}(1 / p-1)}}{\Phi^{1 / 2 p}\left(2^{\left.2 \alpha_{k}\right)}\right.}, & \text { if } j \in\left\{2^{2 \alpha_{k}}, \ldots, 2^{2 \alpha_{k}+1}-1\right\}, k \in \mathbb{N}, \\ 0, & \text { if } j \notin \bigcup_{k=1}^{\infty}\left\{2^{2 \alpha_{k}}, \ldots, 2^{2 \alpha_{k}+1}-1\right\} .\end{cases}
$$

For $n=\sum_{i=1}^{s} 2^{n_{i}}, n_{1}<n_{2}<\cdots<n_{s}$ we denote

$$
\mathbb{A}_{0,2}:=\left\{n \in \mathbb{N}: n=2^{0}+2^{2}+\sum_{i=3}^{s_{n}} 2^{n_{i}}\right\}
$$

Let $2^{2 \alpha_{k}} \leq j \leq 2^{2 \alpha_{k}+1}-1$ and $j \in \mathbb{A}_{0,2}$. Then

$$
R_{j}^{w} f=\frac{1}{l_{j}} \sum_{n=1}^{2^{2 \alpha_{k}-1}} \frac{S_{n} f}{n}+\frac{1}{l_{j}} \sum_{n=2^{2 \alpha_{k}}}^{j} \frac{S_{n} f}{n}:=I+I I .
$$

Let $n<2^{2 \alpha_{k}}$. Then from (3.13), (3.14) and (3.15) we have

$$
\begin{aligned}
\left|S_{n}^{w} f(x)\right| & \leq \sum_{\eta=0}^{k-1} \sum_{v=2^{2 \alpha_{\eta}}}^{2^{2 \alpha_{\eta}+1}-1}\left|\widehat{f}^{w}(v)\right| \leq \sum_{\eta=0}^{k-1} \sum_{v=2^{2 \alpha_{\eta}}}^{2^{2 \alpha_{\eta}+1}-1} \frac{2^{2 \alpha_{\eta}(1 / p-1)}}{\Phi^{1 / 2 p}\left(2^{2 \alpha_{\eta}}\right)} \\
& \leq \sum_{\eta=0}^{k-1} \frac{2^{2 \alpha_{\eta} / p}}{\Phi^{1 / 2 p}\left(2^{2 \alpha_{\eta}}\right)} \leq \frac{2^{2 \alpha_{k-1} / p+1}}{\Phi^{1 / 2 p}\left(2^{\left.2 \alpha_{k-1}\right)}\right.} \leq \frac{1}{128 \alpha_{k}} \frac{2^{2 \alpha_{k}(1 / p-2)}}{\Phi^{1 / 2 p}\left(2^{\left.2 \alpha_{k}\right)}\right.} .
\end{aligned}
$$

Consequently,

$$
\begin{aligned}
|I| & \leq \frac{1}{l_{j}} \sum_{n=1}^{2^{2 \alpha_{k}-1}} \frac{\left|S_{n}^{w} f(x)\right|}{n} \\
& \leq \frac{1}{l_{2^{2 \alpha_{k}}}} \frac{1}{128 \alpha_{k}} \frac{2^{2 \alpha_{k}(1 / p-2)}}{\Phi^{1 / 2 p}\left(2^{2 \alpha_{k}}\right)} \sum_{n=1}^{2^{2 \alpha_{k}-1}} \frac{1}{n} \leq \frac{1}{128 \alpha_{k}} \frac{2^{2 \alpha_{k}(1 / p-2)}}{\Phi^{1 / 2 p}\left(2^{2 \alpha_{k}}\right)}
\end{aligned}
$$

Let $2^{2 \alpha_{k}} \leq n \leq 2^{2 \alpha_{k}+1}-1$. Then we have the following:

$$
\begin{aligned}
S_{n}^{w} f & =\sum_{\eta=0}^{k-1} \sum_{v=2^{2 \alpha_{\eta}}}^{2^{2 \alpha_{\eta}+1}-1} \widehat{f}^{w}(v) w_{v}+\sum_{v=2^{2 \alpha_{k}}}^{n-1} \widehat{f}^{w}(v) w_{v} \\
& =\sum_{\eta=0}^{k-1} \frac{2^{2 \alpha_{\eta}(1 / p-1)}}{\Phi^{1 / 2 p}\left(2^{2 \alpha_{\eta}}\right)}\left(D_{2^{2 \alpha_{\eta}+1}}^{w}-D_{2^{2 \alpha_{\eta}}}^{w}\right)+\frac{2^{2 \alpha_{k}(1 / p-1)}}{\Phi^{1 / 2 p}\left(2^{2 \alpha_{k}}\right)}\left(D_{n}^{w}-D_{2^{2 \alpha_{k}}}^{w}\right) .
\end{aligned}
$$


This gives

$$
\begin{aligned}
I I= & \frac{1}{l_{j}} \sum_{n=2^{2 \alpha_{k}}}^{2^{2 \alpha_{k}+1}} \frac{1}{n}\left(\sum_{\eta=0}^{k-1} \frac{2^{2 \alpha_{\eta}(1 / p-1)}}{\Phi^{1 / 2 p}\left(2^{2 \alpha_{\eta}}\right)}\left(D_{2^{2 \alpha_{\eta}+1}}^{w}-D_{2^{2 \alpha_{\eta}}}^{w}\right)\right) \\
& +\frac{1}{l_{j}} \frac{2^{2 \alpha_{k}(1 / p-1)}}{\Phi^{1 / 2 p}\left(2^{\left.2 \alpha_{k}\right)}\right.} \sum_{n=2^{2 \alpha_{k}}}^{j} \frac{\left(D_{n}^{w}-D_{2^{2 \alpha_{k}}}^{w}\right)}{n} \\
:= & I I_{1}+I I_{2} .
\end{aligned}
$$

Let $x \in I_{2}\left(e_{0}+e_{1}\right) \in I_{0} \backslash I_{1}$. We use well-known equalities for Dirichlet kernels (for details see e.g. [17] and [29]): recall that

$$
D_{2^{n}}^{w}(x)= \begin{cases}2^{n}, & \text { if } x \in I_{n}, \\ 0, & \text { if } x \notin I_{n},\end{cases}
$$

and

$$
D_{n}^{w}=w_{n} \sum_{k=0}^{\infty} n_{k} r_{k} D_{2^{k}}^{w}=w_{n} \sum_{k=0}^{\infty} n_{k}\left(D_{2^{k+1}}^{w}-D_{2^{k}}^{w}\right), \quad \text { for } n=\sum_{i=0}^{\infty} n_{i} 2^{i}
$$

so we can conclude that

$$
D_{n}^{w}(x)= \begin{cases}w_{n}, & \text { if } n \text { is odd number } \\ 0, & \text { if } n \text { is even number. }\end{cases}
$$

Since $\alpha_{0} \geq 2, k \in \mathbb{N}$ we obtain $2 \alpha_{k} \geq 4$, for all $k \in \mathbb{N}$ and if we apply (3.19) we get

$$
I I_{1}=0
$$

and

$$
I I_{2}=\frac{1}{l_{j}} \frac{2^{2 \alpha_{k}(1 / p-1)}}{\Phi^{1 / 2 p}\left(2^{\left.2 \alpha_{k}\right)}\right.} \sum_{n=2^{2 \alpha_{k}-1}}^{(j-1) / 2} \frac{w_{2 n+1}}{2 n+1}=\frac{1}{l_{j}} \frac{2^{2 \alpha_{k}(1 / p-1)} r_{1}}{\Phi^{1 / 2 p}\left(2^{2 \alpha_{k}}\right)} \sum_{n=2^{2 \alpha_{k}-1}}^{(j-1) / 2} \frac{w_{2 n}}{2 n+1}
$$

Let $x \in I_{2}\left(e_{0}+e_{1}\right)$. Then, by the definition of Walsh functions, we get

$$
w_{4 n+2}=r_{1} w_{4 n}=-w_{4 n}
$$

and

$$
\begin{aligned}
\left|I I_{2}\right| & =\frac{1}{l_{j}} \frac{2^{2 \alpha_{k}(1 / p-1)}}{\Phi^{1 / 2 p}\left(2^{2 \alpha_{k}}\right)}\left|\sum_{n=2^{2 \alpha_{k}-1}}^{(j-1) / 2} \frac{w_{2 n}}{2 n+1}\right| \\
& =\frac{1}{l_{j}} \frac{2^{2 \alpha_{k}(1 / p-1)}}{\Phi^{1 / 2 p}\left(2^{2 \alpha_{k}}\right)}\left|\frac{w_{j-1}}{j}+\sum_{n=2^{2 \alpha_{k}-2}+1}^{(j-1) / 4}\left(\frac{w_{4 n-4}}{4 n-3}+\frac{w_{4 n-2}}{4 n-1}\right)\right|
\end{aligned}
$$




$$
\begin{aligned}
& =\frac{1}{l_{j}} \frac{2^{2 \alpha_{k}(1 / p-1)}}{\Phi^{1 / 2 p}\left(2^{\left.2 \alpha_{k}\right)}\right.}\left|\frac{w_{j-1}}{j}+\sum_{n=2^{2 \alpha_{k}-2}+1}^{(j-1) / 4}\left(\frac{w_{4 n-4}}{4 n-3}-\frac{w_{4 n-2}}{4 n-1}\right)\right| \\
& \geq \frac{c}{\log \left(2^{2 \alpha_{k}+1}\right)} \frac{2^{2 \alpha_{k}(1 / p-1)}}{\Phi^{1 / 2 p}\left(2^{\left.2 \alpha_{k}\right)}\right.}\left(\left|\frac{w_{j-1}}{j}\right|-\sum_{n=2^{2 \alpha_{k}-2}+1}^{(j-1) / 4}\left|w_{4 n-4}\right|\left(\frac{1}{4 n-3}-\frac{1}{4 n-1}\right)\right) \\
& \geq \frac{1}{4 \alpha_{k}} \frac{2^{2 \alpha_{k}(1 / p-1)}}{\Phi^{1 / 2 p}\left(2^{\left.2 \alpha_{k}\right)}\right.}\left(\frac{1}{j}-\sum_{n=2^{2 \alpha_{k}-2}+1}^{(j-1) / 4}\left(\frac{1}{4 n-3}-\frac{1}{4 n-1}\right)\right) .
\end{aligned}
$$

By a simple calculation we can conclude that

$$
\begin{aligned}
& \sum_{n=2^{2 \alpha_{k}-2}+1}^{(j-1) / 4}\left(\frac{1}{4 n-3}-\frac{1}{4 n-1}\right) \\
& =\sum_{n=2^{2 \alpha_{k}-2}+1}^{(j-1) / 4} \frac{2}{(4 n-3)(4 n-1)} \\
& \leq \sum_{n=2^{2 \alpha_{k}-2}+1}^{(j-1) / 4} \frac{2}{(4 n-4)(4 n-2)}=\frac{1}{2} \sum_{n=2^{2 \alpha_{k}-2}+1}^{(j-1) / 4} \frac{1}{(2 n-2)(2 n-1)} \\
& \leq \frac{1}{2} \sum_{n=2^{2 \alpha_{k}-2}+1}^{(j-1) / 4} \frac{1}{(2 n-2)(2 n-2)}=\frac{1}{8} \sum_{n=2^{2 \alpha_{k}-2}+1}^{(j-1) / 4} \frac{1}{(n-1)(n-1)} \\
& \leq \frac{1}{8} \sum_{n=2^{2 \alpha_{k}-2}+1}^{(j-1) / 4} \frac{1}{(n-1)(n-2)}=\frac{1}{8} \sum_{l=2^{2 \alpha_{k}-2}+1}^{(j-1) / 4}\left(\frac{1}{n-2}-\frac{1}{n-1}\right) \\
& \leq \frac{1}{8}\left(\frac{1}{2^{2 \alpha_{k}-2}-1}-\frac{4}{j-5}\right) \leq \frac{1}{8}\left(\frac{1}{2^{2 \alpha_{k}-2}-1}-\frac{4}{j}\right) .
\end{aligned}
$$

Since $2^{2 \alpha_{k}} \leq j \leq 2^{2 \alpha_{k}+1}-1$, where $\alpha_{k} \geq 2$, we obtain

$$
\frac{2}{2^{2 \alpha_{k}-4}} \leq \frac{2}{2^{4}-4}=\frac{1}{6}
$$

and

$$
\begin{aligned}
& \left|I I_{2}\right| \geq \frac{1}{4 \alpha_{k}} \frac{2^{2 \alpha_{k}(1 / p-1)}}{\Phi^{1 / 2 p}\left(2^{\left.2 \alpha_{k}\right)}\right.}\left(\frac{1}{j}-\frac{1}{8}\left(\frac{1}{2^{2 \alpha_{k}-2}-1}-\frac{4}{j}\right)\right) \\
& \geq \frac{1}{4 \alpha_{k}} \frac{2^{2 \alpha_{k}(1 / p-1)}}{\Phi^{1 / 2 p}\left(2^{2 \alpha_{k}}\right)}\left(\frac{3}{2 j}-\frac{1}{2^{2 \alpha_{k}+1}-8}\right) \\
& \geq \frac{1}{4 \alpha_{k}} \frac{2^{2 \alpha_{k}(1 / p-1)}}{\Phi^{1 / 2 p}\left(2^{\left.2 \alpha_{k}\right)}\right.}\left(\frac{3}{4} \frac{1}{2^{2 \alpha_{k}}}-\frac{1}{2} \frac{1}{2^{2 \alpha_{k}}-4}\right) \\
& \geq \frac{1}{4 \alpha_{k}} \frac{2^{2 \alpha_{k}(1 / p-1)}}{\Phi^{1 / 2 p}\left(2^{2 \alpha_{k}}\right)}\left(\frac{1}{4} \frac{1}{2^{2 \alpha_{k}}}+\frac{1}{2} \frac{1}{2^{2 \alpha_{k}}}-\frac{1}{2} \frac{1}{2^{2 \alpha_{k}}-4}\right) \\
& =\frac{1}{4 \alpha_{k}} \frac{2^{2 \alpha_{k}(1 / p-1)}}{\Phi^{1 / 2 p}\left(2^{2 \alpha_{k}}\right)}\left(\frac{1}{4} \frac{1}{2^{2 \alpha_{k}}}-\frac{2}{2^{2 \alpha_{k}}\left(2^{2 \alpha_{k}}-4\right)}\right) \\
& \geq \frac{1}{4 \alpha_{k}} \frac{2^{2 \alpha_{k}(1 / p-1)}}{\Phi^{1 / 2 p}\left(2^{\left.2 \alpha_{k}\right)}\right.}\left(\frac{1}{4} \frac{1}{2^{2 \alpha_{k}}}-\frac{1}{6} \frac{1}{2^{2 \alpha_{k}}}\right)
\end{aligned}
$$




$$
\geq \frac{1}{48 \alpha_{k}} \frac{2^{2 \alpha_{k}(1 / p-2)}}{\Phi^{1 / 2 p}\left(2^{2 \alpha_{k}}\right)} \geq \frac{1}{64 \alpha_{k}} \frac{2^{2 \alpha_{k}(1 / p-2)}}{\Phi^{1 / 2 p}\left(2^{\left.2 \alpha_{k}\right)}\right.} .
$$

By combining (3.14), (3.16)-(3.23) for $\in I_{2}\left(e_{0}+e_{1}\right)$ and $0<p<1 / 2$ we find that

$$
\begin{aligned}
\left|R_{j}^{w} f(x)\right| & \geq\left|I I_{2}\right|-\left|I I_{1}\right|-|I| \\
& \geq \frac{1}{64 \alpha_{k}} \frac{2^{2 \alpha_{k}(1 / p-2)}}{\Phi^{1 / 2 p}\left(2^{2 \alpha_{k}}\right)}-\frac{1}{128 \alpha_{k}} \frac{2^{2 \alpha_{k}(1 / p-2)}}{\Phi^{1 / 2 p}\left(2^{\left.2 \alpha_{k}\right)}\right.}=\frac{1}{128 \alpha_{k}} \frac{2^{2 \alpha_{k}(1 / p-2)}}{\Phi^{1 / 2 p}\left(2^{2 \alpha_{k}}\right)} .
\end{aligned}
$$

Hence,

$$
\begin{aligned}
& \left\|R_{j}^{w} f\right\|_{\text {weak- } L_{p}\left(G_{2}\right)}^{p} \\
& \geq \frac{1}{128 \alpha_{k}^{p}} \frac{2^{2 \alpha_{k}(1-2 p)}}{\Phi^{1 / 2}\left(2^{\left.2 \alpha_{k}\right)}\right.} \mu\left\{x \in G_{2}:\left|R_{j}^{w} f\right| \geq \frac{1}{128 \alpha_{k}} \frac{2^{2 \alpha_{k}(1 / p-2)}}{\Phi^{1 / 2 p}\left(2^{\left.2 \alpha_{k}\right)}\right.}\right\}^{1 / p} \\
& \geq \frac{1}{128 \alpha_{k}^{p}} \frac{2^{2 \alpha_{k}(1-2 p)}}{\Phi^{1 / 2}\left(2^{\left.2 \alpha_{k}\right)}\right.} \mu\left\{x \in I_{2}\left(e_{0}+e_{1}\right):\left|R_{j}^{w} f\right| \geq \frac{1}{128 \alpha_{k}} \frac{2^{2 \alpha_{k}(1 / p-2)}}{\Phi^{1 / 2 p}\left(2^{\left.2 \alpha_{k}\right)}\right.}\right\} \\
& \geq \frac{1}{128 \alpha_{k}^{p}} \frac{2^{2 \alpha_{k}(1-2 p)}}{\Phi^{1 / 2}\left(2^{\left.2 \alpha_{k}\right)}\right.}\left(\mu\left(x \in I_{2}\left(e_{0}+e_{1}\right)\right)\right)>\frac{1}{516 \alpha_{k}^{p}} \frac{2^{2 \alpha_{k}(1-2 p)}}{\Phi^{1 / 2}\left(2^{2 \alpha_{k}}\right)} .
\end{aligned}
$$

Moreover,

$$
\begin{aligned}
& \sum_{j=1}^{\infty} \frac{\left\|R_{j}^{w} f\right\|_{\text {weak }-L_{p}\left(G_{2}\right)}^{p} \log ^{p}(j) \Phi(j)}{j^{2-2 p}} \\
& \quad \geq \sum_{\left\{j \in \mathbb{A}_{0,2}: 2^{\left.2 \alpha_{k}<j \leq 2^{2 \alpha_{k}+1}-1\right\}}\right.} \frac{\left\|R_{j}^{w} f\right\|_{\text {weak- } L_{p}}^{p} \log ^{p}(j) \Phi(j)}{j^{2-2 p}} \\
& \geq \frac{c}{\alpha_{k}^{p}} \frac{2^{2 \alpha_{k}(1-2 p)}}{\Phi p^{p / 2}\left(2^{\left.2 \alpha_{k}\right)}\right.} \sum_{\left\{j \in \mathbb{A}_{0,2}: 2^{\left.2 \alpha_{k}<j \leq 2^{2 \alpha_{k}+1}-1\right\}}\right.} \frac{\log ^{p}(j) \Phi(j)}{j^{2-2 p}} \\
& \geq \frac{c \Phi\left(2^{2 \alpha_{k}}\right) \log ^{p}\left(2^{2 \alpha_{k}}\right)}{\alpha_{k}^{p}} \frac{2^{2 \alpha_{k}(1-2 p)}}{\Phi^{1 / 2}\left(2^{\left.2 \alpha_{k}\right)}\right.} \sum_{\left\{j \in \mathbb{A}_{0,2}: 2^{\left.2 \alpha_{k<j} \leq 2^{2 \alpha_{k}+1}-1\right\}}\right.} \frac{1}{j^{2-2 p}} \\
& \geq \Phi^{1 / 2}\left(2^{2 \alpha_{k}}\right) \rightarrow \infty, \quad \text { as } k \rightarrow \infty .
\end{aligned}
$$

The proof is complete.

\section{Final remarks and open problems}

In this section we present some final remarks and open problems, which might be interesting for further research. The first problem reads as follows.

Problem 1 For any $f \in H_{1 / 2}$, is it possible to find strong convergence theorems for Riesz means $R_{m}^{w}$, where $\alpha=w$ or $\alpha=\psi$ ?

Remark 1 Similar problems for Fejér means with respect to Walsh and Vilenkin systems can be found in [2, 4, 40] (see also [45] and [48]). Our method and estimations of Riesz and Fejér kernels (see Lemmas 1 and 2) do not give an opportunity to prove even similar 
strong convergence result as for the case of Fejer means. In particular, for any $f \in H_{1 / 2}$ is it possible to prove the following inequality:

$$
\frac{1}{\log n} \sum_{k=1}^{n} \frac{\left\|R_{k}^{\alpha} f\right\|_{1 / 2}^{1 / 2}}{k} \leq c\|f\|_{H_{1 / 2}}^{1 / 2}, \quad \text { where } \alpha=w \text { or } \alpha=\psi \text { ? }
$$

It is interesting to generalize Theorem 2 for Vilenkin systems.

Problem 2 For $0<p<1 / 2$ and any nondecreasing function $\Phi: \mathbb{N} \rightarrow[1, \infty)$ satisfying the conditions $\lim _{n \rightarrow \infty} \Phi(n)=+\infty$, is it possible to find a martingale $f \in H_{p}\left(G_{m}\right)$ such that

$$
\sum_{n=1}^{\infty} \frac{\log ^{p} n\left\|R_{n}^{\psi} f\right\|_{p}^{p} \Phi(n)}{n^{2-2 p}}=\infty
$$

where $R_{n}^{\psi} f$ denotes the $n$th Riesz logarithmic means with respect to the Vilenkin-Fourier series of $f$ ?

Problem 3 Is it possible to find a martingale $f \in H_{1 / 2}$, such that

$$
\sup _{n \in \mathbb{N}}\left\|R_{n}^{\alpha} f\right\|_{1 / 2}=\infty
$$

where $\alpha=w$ or $\alpha=\psi$ ?

Remark 2 For $0<p<1 / 2$, divergence in the space $L_{p}$ of Riesz logarithmic means with respect to Walsh and Vilenkin systems of martingale $f \in H_{p}$ was already proved in [27].

Problem 4 For any $f \in H_{p}(0<p \leq 1 / 2)$, is it possible to find necessary and sufficient conditions for the indices $k_{j}$ for which

$$
\left\|R_{k j}^{\alpha} f-f\right\|_{H_{p}} \rightarrow 0, \quad \text { as } j \rightarrow \infty
$$

where $\alpha=w$ or $\alpha=\psi$ ?

Remark 3 Similar problem for partial sums and Fejer means with respect to Walsh and Vilenkin systems can be found in Tephnadze [41, 42] and [43].

Problem 5 Is it possible to find necessary and sufficient conditions in terms of the onedimensional modulus of continuity of martingale $f \in H_{p}(0<p \leq 1 / 2)$, for which

$$
\left\|R_{j}^{\alpha} f-f\right\|_{H_{p}} \rightarrow 0, \quad \text { as } j \rightarrow \infty
$$

where $\alpha=w$ or $\psi$ ?

Remark 4 Approximation properties of some summability methods in the classical and real Hardy spaces were considered by Oswald [24], Kryakin and Trebels [18], Storoienko [32, 33] and for martingale Hardy spaces in Fridli, Manchanda and Siddiqi [9] (see also [7, 8]), Nagy [20-22], Tephnadze [41-43]. 


\section{Acknowledgements}

The research was supported by Shota Rustaveli National Science Foundation grant FR-19-676. We thank the reviewers for some important remarks, which have improved the final version of the paper.

Funding

Not applicable.

\section{Availability of data and materials}

Not applicable.

\section{Competing interests}

The authors declare that they have no competing interests.

\section{Authors' contributions}

All the authors contributed equally and significantly in writing this paper. All the authors read and approved the final manuscript.

\section{Authors' information}

DL is professor of Mathematics at UiT The Arctic University of Norway. LEP is professor of Mathematics at UiT The Arctic University of Norway. He was previously President of the Swedish Mathematical Society. GTe is Associate Professor at the University of Georgia. GTu is PhD student at the University of Georgia and UiT The Arctic University of Norway

\section{Author details}

${ }^{1}$ UiT The Arctic University of Norway, Narvik, Norway. ${ }^{2}$ Department of Mathematics and Computer Science, Karlstad University, Karlstad, Sweden. ${ }^{3}$ School of Science and Technology, The University of Georgia, Tbilisi, Georgia. ${ }^{4}$ School of IT, Engineering and Mathematics, The University of Georgia, Tbilisi, Georgia.

\section{Publisher's Note}

Springer Nature remains neutral with regard to jurisdictional claims in published maps and institutional affiliations.

Received: 27 November 2019 Accepted: 11 March 2020 Published online: 23 March 2020

\section{References}

1. Agaev, G.N., Vilenkin, N.Y., Dzhafarly, G.M., Rubinshtein, A.I:: Multiplicative systems of functions and harmonic analysis on zero-dimensional groups, Baku, Ehim (1981) (in Russian)

2. Baramidze, L., Persson, L.E., Tephnadze, G., Wall, P.: Sharp $H_{p}-L_{p}$ type inequalities of weighted maximal operators of Vilenkin-Nörlund means and its applications. J. Inequal. Appl. (2016). https://doi.org/10.1186/s13660-016-1182-1

3. Blahota, I., Gát, G.: Norm summability of Nörlund logarithmic means on unbounded Vilenkin groups. Anal. Theory Appl. 24(1), 1-17 (2008)

4. Blahota, I., Tephnadze, G.: Strong convergence theorem for Vilenkin-Fejér means. Publ. Math. (Debr.) 85(1-2) 181-196 (2014)

5. Coifman, R., Weiss, G.: Extensions of Hardy spaces and their use in analysis. Bull. Am. Math. Soc. 83(4), $569-645$ (1977)

6. Fefferman, Ch., Stein, E.M.: HP spaces of several variables. Acta Math. 129, 137-193 (1972)

7. Fridli, S:: Approximation by Vilenkin-Fourier sums. Acta Math. Hung. 47(1-2), 33-44 (1986)

8. Fridli, S.: On the rate of convergence of Cesàro means of Walsh-Fourier series. J. Approx. Theory 76(1), 31-53 (1994)

9. Fridli, S., Manchanda, P., Siddiqi, A.H.: Approximation by Walsh-Nörlund means. Acta Sci. Math. 74(3-4), 593-608 (2008)

10. Fujii, N.J.: A maximal inequality for $H_{1}$ functions on the generalized Walsh-Paley group. Proc. Am. Math. Soc. 77, 111-116 (1979)

11. Gát, G.: Investigations of certain operators with respect to the Vilenkin systems. Acta Math. Hung. 61(1-2), 131-149 (1993)

12. Gát, G.: Cesáro means of integrable functions with respect to unbounded Vilenkin systems. J. Approx. Theory 124(1), 25-43 (2003)

13. Gát, G., Nagy, K.: On the logarithmic summability of Fourier series. Georgian Math. J. 18(2), 237-248 (2011)

14. Goginava, U.: The maximal operator of Marcinkiewicz-Fejér means of the d-dimensional Walsh-Fourier series. East J. Approx. 12(3), 295-302 (2006)

15. Goginava, U.: Maximal operators of logarithmic means of one-dimensional Walsh-Fourier series. Rend. Circ. Mat. Palermo 82, 345-357 (2010)

16. Goginava, U., Nagy, K.: On the maximal operator of Walsh-Kaczmarz-Fejér means. Czechoslov. Math. J. 61(136), 673-686 (2011)

17. Golubov, B., Efimov, A., Skvortsov, V.: Walsh Series and Transformations. Kluwer Academic, Dordrecht (1991) 1991

18. Kryakin, Y., Trebels, W.: $q$-Moduli of continuity in $H_{p}(D), p>0$, and a inequality of Hardy and Littlewood. J. Approx. Theory 115, 238-259 (2002)

19. Later, R.H.: A characterization of $H_{p}\left(R^{n}\right)$ in terms of atoms. Stud. Math. 62, 93-101 (1978)

20. Nagy, K.: Approximation by Nörlund means of quadratical partial sums of double Walsh-Fourier series. Anal. Math. 26, 299-319 (2010)

21. Nagy, K.: On the maximal operator of Walsh-Marcinkiewicz means. Publ. Math. (Debr.) 78(3-4), 633-646 (2011)

22. Nagy, K.: Approximation by Cesàro means of negative order of double Walsh-Kaczmarz-Fourier series. Tohoku Math. J. 64, 317-331 (2012)

23. Neveu, J.: Discrete-Parameter Martingales. North-Holland Mathematical Library, vol. 10. North-Holland, Amsterdam (1975) 
24. Oswald, P.: On some approximation properties of real Hardy spaces $(0<p \leq 1)$. J. Approx. Theory 40, 45-65 (1984)

25. Pal, J., Simon, P.: On a generalization of the concept of derivate. Acta Math. Hung. 29, 155-164 (1977)

26. Persson, L.-E., Ragusa, M.A., Samko, N., Wall, P.: Commutators of Hardy operators in vanishing Morrey spaces. In: AlP Conference Proceedings, vol. 1493, pp. 859-866 (2012)

27. Persson, L.E., Tephnadze, G., Wall, P.: On the maximal operators of Vilenkin-Nörlund means. J. Fourier Anal. Appl. 21(1), 76-94 (2015)

28. Schipp, F.: Certain rearrangements of series in the Walsh series. Mat. Zametki 18, 193-201 (1975)

29. Schipp, F., Wade, W.R., Simon, P., Pál, J.: Walsh Series, an Introduction to Dyadic Harmonic Analysis. Hilger, Bristol (1990)

30. Simon, P.: Investigations with respect to the Vilenkin system. Ann. Univ. Sci. Bp. Rolando Eötvös Nomin., Sect. Math. 28, 87-101 (1985)

31. Simon, P., Weisz, F.: Weak inequalities for Cesáro and Riesz summability of Walsh-Fourier series. J. Approx. Theory 151, $1-19$ (2008)

32. Storozenko, E.A.: On approximation of functions of the class $H^{p}, 0<p<1$. Soobsc. Akad. Nauk Gruz., SSR $88,45-48$ (1977) Russian

33. Storozenko, E.A.: Approximation of functions of the class $H^{p}, 0<p<1$. Mat. Sb. 105(149), 601-621 (1978) Russian

34. Tephnadze, G.: Fejér means of Vilenkin-Fourier series. Studia Sci. Math. Hung. 49(1), 79-90 (2012)

35. Tephnadze, G.: On the maximal operator of Vilenkin-Fejér means. Turk. J. Math. 37, 308-318 (2013)

36. Tephnadze, G.: On the maximal operators of Vilenkin-Fejér means on Hardy spaces. Math. Inequal. Appl. 16(1), 301-312(2013)

37. Tephnadze, G.: On the maximal operators of Walsh-Kaczmarz-Fejér means. Period. Math. Hung. 67(1), 33-45 (2013)

38. Tephnadze, G.: Strong convergence theorems of Walsh-Fejér means. Acta Math. Hung. 142(1), 244-259 (2014)

39. Tephnadze, G.: On the maximal operators of Riesz logarithmic means of Vilenkin-Fourier series. Studia Sci. Math. Hung. 51(1), 105-120 (2014)

40. Tephnadze, G.: Strong convergence theorems of Walsh-Fejér means. Acta Math. Hung. 142(1), 244-259 (2014)

41. Tephnadze, G.: On the partial sums of Walsh-Fourier series. Colloq. Math. 141(2), 227-242 (2015)

42. Tephnadze, G.: On the convergence of Fejér means of Walsh-Fourier series in the space $H_{p}$. J. Contemp. Math. Anal. 51(2), 51-63 (2016)

43. Tephnadze, G.: On the convergence of partial sums with respect to Vilenkin system on the martingale Hardy spaces J. Contemp. Math. Anal. 53(5), 294-306 (2018)

44. Torchinsky, A.: Real-Variable Methods in Harmonic Analysis. Dover Books on Mathematics (2008)

45. Tutberidze, G.: A note on the strong convergence of partial sums with respect to Vilenkin system. J. Contemp. Math. Anal. (in press)

46. Weisz, F.: Martingale Hardy Spaces and Their Application in Fourier Analysis. Springer, Berlin (1994)

47. Weisz, F.: Cesáro summability of one- and two-dimensional Walsh-Fourier series. Anal. Math. 22(3), 229-242 (1996)

48. Weisz, F.: Strong convergence theorems for two-parameter Walsh-Fourier and trigonometric-Fourier series. Stud. Math. 117(2), 173-194 (1996)

49. Weisz, F.: Summability of Multi-Dimensional Fourier Series and Hardy Space. Kluwer Academic, Dordrecht (2002)

50. Weisz, F.: Q-summability of Fourier series. Acta Math. Hung. 103(1-2), 139-175 (2004)

51. Wilson, J.M.: A simple proof of the atomic decomposition for $H_{p}\left(R_{n}\right) 0<p \leq 1$. Stud. Math. 74, 25-33 (1982)

\section{Submit your manuscript to a SpringerOpen ${ }^{\circ}$ journal and benefit from:}

- Convenient online submission

- Rigorous peer review

- Open access: articles freely available online

- High visibility within the field

- Retaining the copyright to your article

Submit your next manuscript at springeropen.com 